\title{
Main pulmonary artery area limits exercise capacity in patients long-term after arterial switch operation
}

\author{
Vivan J. M. Baggen, MD, ${ }^{\mathrm{a}, \mathrm{b}}$ Mieke M. P. Driessen, MD, ${ }^{\mathrm{b}, \mathrm{c}}$ Folkert J. Meijboom, MD, PhD, ${ }^{\mathrm{b}}$ \\ Gertjan Tj. Sieswerda, MD, PhD, ${ }^{b}$ Nicolaas J. G. Jansen, MD, PhD, ${ }^{d}$ Sebastiaan W. H. van Wijk, MD, \\ Pieter A. Doevendans, MD, PhD, FESC, ${ }^{\mathrm{b}}$ Tim Leiner, MD, PhD, EBCR, ${ }^{\mathrm{e}}$ Paul H. Schoof, MD, PhD, ${ }^{\mathrm{f}}$ \\ Tim Takken, MSc, PhD, ${ }^{\mathrm{g}}$ and Johannes M. P. J. Breur, MD, $\mathrm{PhD}^{\mathrm{h}}$
}

\begin{abstract}
Objectives: Despite excellent survival in patients after the arterial switch operation, reintervention is frequently required and exercise capacity is decreased in a substantial number of patients. This study relates right-sided imaging features in patients long-term after the arterial switch operation to exercise capacity and ventilatory efficiency to investigate which lesions are functionally important.
\end{abstract}

Methods: Patients operated in the UMC Utrecht, the Netherlands (1976-2001) and healthy controls underwent cardiac magnetic resonance imaging and cardiopulmonary exercise testing within 1 week. We measured main, left, and right pulmonary artery cross-sectional areas, pulmonary blood flow distribution, peak oxygen uptake, and minute ventilation relative to carbon dioxide elimination.

Results: A total of 71 patients (median age, 20 [12-35] years, 73\% were male) and 21 healthy controls (median age, 26 [21-35] years, $48 \%$ were male) were included. Main, left, and right pulmonary artery areas were decreased compared with controls (190 vs $269 \mathrm{~mm}^{2} / \mathrm{m}^{2}, 59$ vs $157 \mathrm{~mm}^{2} / \mathrm{m}^{2}$, 98 vs $139 \mathrm{~mm}^{2} / \mathrm{m}^{2}$, respectively, all $P<.001)$; however, pulmonary blood flow distribution was comparable $(P=.722)$. Peak oxygen uptake and minute ventilation relative to carbon dioxide elimination were $88 \% \pm 20 \%$ and $23.7 \pm 3.8$, respectively, with $42 \%$ and $1 \%$ of patients demonstrating abnormal results $(\leq 84 \%$ and $\geq 34$, respectively). The main pulmonary artery area significantly correlated with peak oxygen uptake $(r=0.401, P=.001)$ and pulmonary blood flow distribution with minute ventilation relative to carbon dioxide elimination ( $r=-0.329, P=.008)$. Subanalysis $(<18,18-25,>25$ years $)$ showed that the main pulmonary artery area was smaller in older age groups. In multivariable analysis, the main pulmonary artery area was independently associated with peak oxygen uptake $(P=.032)$.

Conclusions: In adult patients after the arterial switch operation, narrowing of the main pulmonary artery is a common finding and is the main determinant of limitation in functional capacity, rather than pulmonary branch stenosis. (J Thorac Cardiovasc Surg 2015;150:918-25)

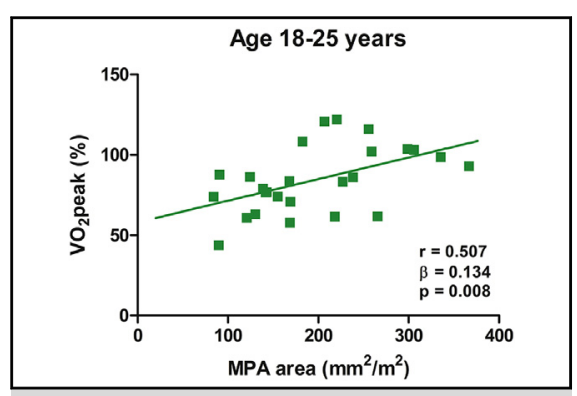

Association of MPA area with exercise capacity in patients aged 18 to 25 years. MPA, Main pulmonary artery; $\mathrm{VO}_{2}$ peak $\%$, peak oxygen uptake $(\%$ of predicted)

\section{Central Message}

In adult patients after the arterial switch operation, narrowing of the MPA is the main determinant of limitation in functional capacity.

\section{Perspective}

In this study, the importance of MPA narrowing as a determinant for exercise capacity is highlighted. This could imply that during the arterial switch operation, optimization of MPA size is important for functional outcomes. Moreover, this suggests that the long-term follow-up of patients after the arterial switch operation should focus on the presence or progression of MPA stenosis.

See Editorial Commentary page 926.

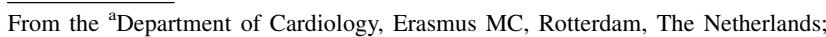
Departments of ${ }^{\mathrm{b}}$ Cardiology and ${ }^{\mathrm{e}}$ Radiology, UMC Utrecht, Utrecht, The Netherlands; ' ICIN-Netherlands Heart Institute, Utrecht, The Netherlands; Departments of ${ }^{\mathrm{d}}$ Pediatric Intensive Care, ${ }^{\mathrm{f}}$ Pediatric Cardiothoracic Surgery, and hPediatric Cardiology, Wilhelmina Children's Hospital, UMC Utrecht, Utrecht, The Netherlands; and ${ }^{\mathrm{g}}$ Department of Pediatric Physical Therapy and Exercise Physiology, Child Development and Exercise Center, Wilhelmina Children's Hospital, UMC Utrecht, Utrecht, The Netherlands.

V.J.M.B. and M.M.P.D. share first authorship.
Received for publication May 30, 2015; revisions received July 22, 2015; accepted for publication July 29, 2015; available ahead of print Aug 28, 2015.

Address for reprints: Johannes M.P.J. Breur, MD, PhD, Department of Pediatric Cardiology, Wilhelmina Children's Hospital, UMC Utrecht, Room KG.01.319.0, PO Box 85500,3508 GA, Utrecht, The Netherlands (E-mail: h.breur@umcutrecht.nl). $0022-5223 / \$ 36.00$

Copyright (C) 2015 by The American Association for Thoracic Surgery http://dx.doi.org/10.1016/j.jtcvs.2015.07.101 


\begin{tabular}{|c|c|}
\hline \multicolumn{2}{|c|}{ Abbreviations and Acronyms } \\
\hline CMR & $=$ cardiac magnetic resonance \\
\hline LPA & $=$ left pulmonary artery \\
\hline MPA & $=$ main pulmonary artery \\
\hline MRA & $=$ magnetic resonance angiography \\
\hline PBF & $=$ pulmonary blood flow \\
\hline RPA & $=$ right pulmonary artery \\
\hline RV & $=$ right ventricle/right ventricular \\
\hline RVOT & $=$ right ventricular outflow tract \\
\hline 3D & $=3$-dimensional \\
\hline $\mathrm{VE} / \mathrm{VCO}_{2}$ slop & $\begin{aligned}= & \text { minute ventilation relative to } \\
& \text { carbon dioxide elimination }\end{aligned}$ \\
\hline $\mathrm{VO}_{2}$ peak $\%$ & $\begin{array}{l}=\text { peak oxygen uptake }(\% \text { of } \\
\text { predicted) }\end{array}$ \\
\hline
\end{tabular}

Transposition of the great arteries is the most common cyanotic congenital heart defect, occurring in 4.7 of 10.000 newborns. ${ }^{1}$ The current treatment of choice is neonatal arterial switch operation, as described in 1976 by Jatene and colleagues. $^{2}$

Follow-up studies show excellent results with low perioperative mortality and high 25-year survival $(>95 \%)$. However, the cumulative risk of reintervention increases up to $25 \%$ in adult patients. ${ }^{3,4}$ Most of these patients have supravalvular neopulmonary artery or pulmonary branch stenosis and undergo balloon dilatation or stenting by catheter intervention or surgical pulmonary artery reconstruction. ${ }^{4}$ Apart from reintervention, longterm follow-up studies show that exercise capacity is decreased in a significant subset of patients. ${ }^{5-7}$ Reduced exercise capacity has been associated with right-sided obstructive lesions. ${ }^{6,7}$ Still, long-term follow-up data are limited, and defined management strategies for subclinical anatomic or physiologic abnormalities are lacking. ${ }^{8}$

This study compared patients long-term after the arterial switch operation with healthy controls, focusing on right ventricular (RV) function, pulmonary artery and branch cross-sectional areas, pulmonary branch relative area change, and pulmonary blood flow (PBF) distribution. Second, these imaging features were related to exercise capacity and ventilatory efficiency to determine which lesions are functionally important and therefore potentially amenable to reintervention.

\section{MATERIALS AND METHODS \\ Study Population}

We performed a cross-sectional cohort study between August 2011 and February 2014. All patients who underwent an arterial switch operation in our center who were aged more than 12 years (1976-2001) were approached. Patients prospectively underwent cardiac magnetic resonance (CMR) imaging, echocardiography, and cardiopulmonary exercise testing within 1 week, without any change in clinical condition. Healthy control subjects (aged 18-35 years) underwent CMR and echocardiography with the same study protocol. Exercise testing was not performed in healthy controls because reference values are well established. ${ }^{9}$ The institutional review committee of the University Medical Center Utrecht approved this study. Informed consent was obtained from all patients, parents if aged less than 18 years of age, and healthy controls. Patient characteristics were obtained from the patient chart. Patients with a homograft, signs of myocardial ischemia during the exercise test, or claustrophobia were excluded.

\section{Cardiac Magnetic Resonance Acquisition}

Patients and controls were scanned according to a predefined imaging protocol without anesthesia or sedation. A 1.5-T system (Ingenia R4.1.2, Philips Healthcare, Best, The Netherlands) was used with a dedicated chest phased-array parallel imaging-capable surface coil with a maximum of 36 active elements.

Steady-state free precession cine images were acquired in various orientations (short axis, 4-chamber and 2-chamber long axis, right and left ventricular outflow tract views in 2 planes) during repeated end-expiratory breath holds. Multi-slice cine short-axis acquisition was planned from the apex to well above the tricuspid and mitral valve: TR/TE 3.4/1.69 ms, voxel size $1.25 \times 1.25 \times 8 \mathrm{~mm}$, flip angle $90^{\circ}$, matrix $192 \times 171 \mathrm{~mm}, 30$ frames/cycle.

Gadolinium-enhanced magnetic resonance angiography (MRA) images of the pulmonary vasculature were performed with non-electrocardiographygated time-resolved 3-dimensional (3D) spoiled gradient echo sequence: TR/TE 5.2/1.49, voxel size $0.94 \times 0.94 \times 2 \mathrm{~mm}, 60$ slices, field of view 300 , matrix $308 \times 125$, keyhole percentage $25 \%, 3$ dynamics, keyhole scan time 2.8 seconds. Gadopentetate dimeglumine $(0.2 \mathrm{~mL} / \mathrm{kg}$, injection rate $1.5 \mathrm{~mL} / \mathrm{s})$ was injected intravenously followed by saline flush $(25 \mathrm{~mL})$.

Quantitative through-plane flow through the left pulmonary artery (LPA) and right pulmonary artery (RPA) was measured with a retrospectively electrocardiography gated, velocity encoded phase-contrast sequence: TR/TE $5.2 / 3.1 \mathrm{~ms}$, voxel size $2.5 \times 2.5 \times 8 \mathrm{~mm}$, flip angle 12 , field of view 320 , matrix $128 \times 100$, temporal resolution 20 frames/ cycle. ${ }^{10}$ In case of aliasing, the sequence was rescanned at a higher velocity encoding range. LPA and RPA flow measurements were performed on the narrowest points identified on scout, black-blood, and MRA images.

\section{Cardiac Magnetic Resonance Analysis}

RV volumetric analysis was performed by manual tracing of endocardial and epicardial contours, using Qmass MR Research edition (version 7.4, Medis, Leiden, The Netherlands), with a previously described $\mathrm{RV}$ analysis protocol. ${ }^{11}$ Trabeculae and papillary muscles were selected and excluded from the blood volume using semiautomatic thresholdbased segmentation software, which is based on the signal intensity distribution of the voxels. ${ }^{12}$

Diameters and areas of the main pulmonary artery (MPA), LPA, and RPA were measured on 3D MRA images in OsiriX Imaging Software for MacOS 10.7 (version 5.5.2, 32-bit, Pixmeo, Geneva, Switzerland). Narrowest vessel lumina were selected in 2 perpendicular views to obtain the smallest crosssectional area in the third view. The full width at half maximum method was used to distinguish vessel from surrounding tissue. ${ }^{13}$

Velocity-encoded CMR data were analyzed using QFlow (version 5.6, Medis, Leiden, The Netherlands). Contours were manually traced for the LPA and RPA lumina in all phases. In this way, maximal and minimal areas of the pulmonary branches were obtained. Flow data were determined with a velocity analysis of each voxel in all phases. One researcher performed all CMR analyses and was blinded for the results of the cardiopulmonary exercise test.

\section{Calculation of Right-Sided Imaging Features}

All volumetric data were indexed for body surface area, calculated by the Dubois formula. Pulmonary branch relative area change was calculated by (maximal area - minimal area)/minimal area $\times 100 \%$. To express the 
degree of unilateral pulmonary branch stenosis, relative branch area was calculated by the smallest pulmonary branch area/(LPA + RPA area) $\times 100 \%$. Significant unilateral pulmonary branch stenosis was defined as a relative branch area of less than $30 \%$. PBF distribution was calculated by relative effective stroke volume (forward flow - backward flow per heart beat) to the left and right lung: LPA (or RPA) effective stroke volume/(LPA + RPA effective stroke volume) $\times 100 \%$. Abnormal PBF distribution was defined as a greater than $15 \%$ point deviation from normal (45:55 L:R, thus $<30 \%$ LPA relative flow or $<40 \%$ RPA relative flow).

\section{Echocardiography}

Doppler transthoracic echocardiography was performed on a Toshiba Artida (Toshiba, Tokyo, Japan) with a 5-MHz transducer. The greatest continuous-wave velocity of the tricuspid regurgitation jet was used to express RV systolic pressure, using the Bernoulli equation $\left(4 \mathrm{TR}^{2}\right)$. The greatest continuous-wave velocity measured across the pulmonary valve was used as an index of RV outflow tract (RVOT) obstruction. ${ }^{6}$

\section{Exercise Testing}

Patients performed a cardiopulmonary exercise test using an electronically braked cycle ergometer (Lode Corrival, Lode BV, Groningen, The Netherlands). Before exercise, forced expiratory volume in the first second was measured. After a rest period of 3 minutes and 3 minutes of unloaded cycling, the work rate was increased in a ramp-like protocol with 15,20 , or 25 Watts per minute. It was aimed to complete the test within 8 to 12 minutes. ${ }^{14}$ Maximum effort was defined as a peak respiratory exchange ratio of greater than 1.0 for children and greater than 1.1 for adults. Indications for premature test termination are provided by the American Thoracic Society/American College of Chest Physicians. ${ }^{9}$

During exercise, oxygen uptake $\left(\mathrm{VO}_{2}\right)$, carbon dioxide elimination $\left(\mathrm{VCO}_{2}\right)$, and minute ventilation (VE) were recorded using a computerized breath-by-breath analyzer (ZAN 600, ZAN Me $\beta$ geräte GmbH, Accuramed BVBA, Herk-de-Stad, Belgium). Respiratory exchange ratio was calculated by $\mathrm{VCO}_{2} / \mathrm{VO}_{2}$ and breathing reserve by $(1-$ peak $\mathrm{VE} /$ $\left.\left[\mathrm{FEV}_{1} \times 40\right]\right) \times 100 \%$. Peak oxygen uptake $\left(\mathrm{VO}_{2}\right.$ peak $\left.\%\right)$ was expressed as percentage of the predicted value, based on age, sex, height, and body weight. ${ }^{15}$ Ventilatory efficiency (minute ventilation relative to carbon dioxide elimination $\left[\mathrm{VE} / \mathrm{VCO}_{2}\right.$ slope]) was calculated using linear regression, up to the respiratory compensation point. Peak heart rate $85 \%$ or less, $\mathrm{VO}_{2}$ peak $84 \%$ or less, and $\mathrm{VE} / \mathrm{VCO}_{2}$ slope 34 or greater were considered abnormal. ${ }^{9}$ Specific reference values for children were applied in patients aged 12 to 18 years. ${ }^{16}$

\section{Reproducibility}

Intra- and interobserver reproducibility of RV function analysis were previously assessed and published. ${ }^{11}$ In addition, we assessed reproducibility of 3D MRA analysis by blinded reanalysis of 10 random datasets by a second observer.

\footnotetext{
Statistical Analysis

For all variables, the distribution was tested using the Shapiro-Wilk test. Continuous data were expressed as mean value \pm standard deviation or median (interquartile range) as appropriate. Depending on the distribution of data, the independent-samples $t$ test or the Mann-Whitney $U$ test was used to compare groups. For categoric data, the chi-square test was used. Associations were evaluated using Pearson or Spearman's rho (r) coefficient as appropriate.

For the subanalysis, we could not define clear changes in surgical strategies over time. Therefore, we chose to define 3 age groups $(<18$, $18-25,>25$ years) based on approximately equal years and patients per subgroup. Subanalysis for 3 predefined age groups was performed using chi-square Mantel-Haenszel tests and linear regression for dichotomous and continuous outcomes, respectively.
}

Associations were adjusted for age, sex, and operative characteristics using multivariable linear regression. Reproducibility was tested with intraclass correlation coefficients and paired-samples $t$ test. Mean relative differences were calculated with (Obs1 - Obs2)/([Obs1 + Obs2]/ 2) $\times 100 \%$. All data analysis was performed in IBM SPSS statistics (version 20.0, IBM, Chicago, Ill).

\section{RESULTS \\ Study Population}

Eighty-six perioperative survivors were approached, of whom 74 participated. Three patients with a homograft were excluded, because this predisposes to obstruction and decreased exercise capacity. None of the patients had ST-T-segment dynamics typical of myocardial ischemia. The remaining 71 patients represented the cohort as analyzed in the present study (median age, 20.1 [14.5-26.1] years; range, 12-35, 52 male, 73\%). Exercise testing was performed in 69 patients. Eight patients $(11 \%)$ underwent pulmonary artery banding, and 48 patients $(68 \%)$ underwent the Rashkind procedure. The median age at arterial switch operation was 7 (5-20) days (range, 0-755 days). The Lecompte maneuver was performed in 63 patients $(89 \%) .{ }^{17}$ Ventricular septal defect was surgically closed in 20 patients $(28 \%)$, of whom 2 had Taussig-Bing anomaly; aortic coarctation was repaired in 5 patients $(7 \%)$. Eleven patients $(16 \%)$ underwent 1 or multiple RVOT reinterventions, of whom $7(10 \%)$ underwent a surgical procedure. Twenty-one healthy controls were included (median age, 26.4 [23.2-29.9] years; range, 21-35, 10 male, $48 \%$ ).

\section{Patients After Arterial Switch Operation Compared With Healthy Controls}

Doppler echocardiography and CMR measurements in patients after the arterial switch operation are compared with healthy controls in Table 1. All measured cross-sectional areas of the pulmonary tree (MPA, LPA, and RPA) were significantly and substantially reduced in patients $(P<.001)$. Significant unilateral branch stenosis (relative branch area of $<30 \%$ ) was present in 26 of 70 patients with available 3D MRA data (37\%). Abnormal PBF distribution was present in only 5 of 66 patients with representative flow data $(7 \%)$, of whom 4 had less than $30 \%$ relative LPA flow and 1 had less than $40 \%$ relative RPA flow. PBF distribution and smallest relative and absolute pulmonary branch flow in patients were not significantly different from those in controls $(P=.722$, $P=.444$, and $P=.210$, respectively).

LPA and RPA relative area change did not significantly differ from controls; however, the range of data distribution was larger. The maximal flow velocities across the pulmonary valve (echocardiography) and pulmonary branches (CMR phase-contrast) were increased compared with controls $(P<.001)$. 
TABLE 1. Patients after arterial switch operation compared with healthy controls

\begin{tabular}{|c|c|c|c|}
\hline & $\begin{array}{l}\text { Patients } \\
(\mathrm{n}=\mathbf{7 1})\end{array}$ & $\begin{array}{l}\text { Controls } \\
(\mathbf{n}=\mathbf{2 1})\end{array}$ & $\begin{array}{c}P \\
\text { value }\end{array}$ \\
\hline Age, $y^{*}$ & $20.1(14.5-26.1)$ & $26.4(23.2-29.9)$ & $<.001$ \\
\hline Sex, male $\mathrm{n}(\%) \dagger$ & $52(73)$ & $10(48)$ & .028 \\
\hline $\mathrm{RV}$ systolic pressure, $\mathrm{mm} \mathrm{Hg}$ & $29 \pm 10$ & - & - \\
\hline $\begin{array}{l}\text { Maximal velocity across } \\
\text { pulmonary valve, } \mathrm{cm} / \mathrm{s}\end{array}$ & $207 \pm 68$ & $107 \pm 15$ & $<.001$ \\
\hline \multicolumn{4}{|l|}{ RV function } \\
\hline $\begin{array}{l}\text { RV end-diastolic volume, } \\
\mathrm{mL} / \mathrm{m}^{2} *\end{array}$ & $95(85-104)$ & $104(91-116)$ & .014 \\
\hline $\begin{array}{l}\text { RV end-systolic volume, } \\
\mathrm{mL} / \mathrm{m}^{2} *\end{array}$ & $41(35-48)$ & $50(45-58)$ & .003 \\
\hline $\begin{array}{l}\text { RV stroke volume, } \\
\mathrm{mL} / \mathrm{m}^{2}\end{array}$ & $53 \pm 9$ & $56 \pm 9$ & .160 \\
\hline $\mathrm{RV}$ ejection fraction, $\%$ & $55 \pm 5$ & $53 \pm 4$ & .048 \\
\hline $\mathrm{RV}$ mass, $\mathrm{g} / \mathrm{m}^{2 *}$ & $30(27-35)$ & $23(21-26)$ & $<.001$ \\
\hline \multicolumn{4}{|l|}{$\begin{array}{l}\text { Pulmonary artery cross- } \\
\text { sectional areas }\end{array}$} \\
\hline MPA area, $\mathrm{mm}^{2} / \mathrm{m}^{2} *$ & $190(137-238)$ & $269(242-301)$ & $<.001$ \\
\hline LPA area, $\mathrm{mm}^{2} / \mathrm{m}^{2} *$ & $59(41-86)$ & $157(141-186)$ & $<.001$ \\
\hline $\mathrm{RPA}$ area, $\mathrm{mm}^{2} / \mathrm{m}^{2 *}$ & $98(63-147)$ & $139(123-175)$ & $<.001$ \\
\hline $\mathrm{LPA}+\mathrm{RPA}$ area, $\mathrm{mm}^{2} / \mathrm{m}^{2} *$ & $175(109-228)$ & $300(272-343)$ & $<.001$ \\
\hline $\begin{array}{l}\text { Smallest pulmonary branch } \\
\text { area, } \mathrm{mm}^{2} / \mathrm{m}^{2} *\end{array}$ & $51(37-77)$ & $139(123-169)$ & $<.001$ \\
\hline \multicolumn{4}{|l|}{ Qflow measurements } \\
\hline $\begin{array}{l}\text { LPA relative area } \\
\text { change, } \% *\end{array}$ & $43(31-61)$ & $42(35-53)$ & .754 \\
\hline $\begin{array}{l}\text { RPA relative area } \\
\text { change, } \% *\end{array}$ & $56(40-66)$ & $51(45-65)$ & .966 \\
\hline LPA:RPA flow, $\%$ & $45: 55 \pm 8$ & $45: 55 \pm 4$ & .722 \\
\hline $\begin{array}{l}\text { Smallest pulmonary branch } \\
\text { flow, } \% *\end{array}$ & $44(39-47)$ & $46(41-47)$ & .444 \\
\hline $\begin{array}{l}\text { Smallest pulmonary branch } \\
\text { flow, } \mathrm{mL} / \mathrm{m}^{2 *}\end{array}$ & $21(19-25)$ & $23(21-26)$ & .210 \\
\hline $\begin{array}{l}\text { LPA peak flow velocity, } \\
\mathrm{cm} / \mathrm{s}^{*}\end{array}$ & $168(139-204)$ & $73(66-82)$ & $<.001$ \\
\hline $\begin{array}{l}\text { RPA peak flow velocity, } \\
\mathrm{cm} / \mathrm{s}^{*}\end{array}$ & $166(140-207)$ & $88(77-97)$ & $<.001$ \\
\hline $\begin{array}{l}\text { LPA regurgitation } \\
\text { fraction, } \% *\end{array}$ & $2.7(0.6-10.0)$ & $3.0(2.2-7.0)$ & .565 \\
\hline $\begin{array}{l}\text { RPA regurgitation } \\
\text { fraction, } \% *\end{array}$ & $5.8(1.6-8.5)$ & $1.7(0.6-2.7)$ & .001 \\
\hline
\end{tabular}

$R V$, Right ventricular; $M P A$, main pulmonary artery; $L P A$, left pulmonary artery; $R P A$, right pulmonary artery. *Median (interquartile range) and Mann-Whitney $U$ test. $\dagger \mathrm{n}(\%)$ and chi-square test; other mean \pm standard deviation and independentsamples $t$ test.

\section{Cardiopulmonary Exercise Testing}

Exercise testing results are presented in Table 2. Patients achieved a median peak heart rate of $98 \%$ of predicted (interquartile range, 94-102); 4 of 69 patients (6\%) had an inadequate increase of heart rate during exercise (range, $71 \%-83 \%$ of predicted). Mean $\mathrm{VO}_{2}$ peak $\%$ was $88 \% \pm 20 \%$, and 29 patients $(42 \%)$ were demonstrated abnormal results $(69 \% \pm 11 \%)$. Mean $\mathrm{VE} / \mathrm{VCO}_{2}$ slope
TABLE 2. Exercise testing results of patients after arterial switch operation $(\mathbf{n}=69)$

\begin{tabular}{lc}
\hline \multicolumn{1}{c}{ Variable } & $\begin{array}{c}\text { Mean } \pm \text { SD or } \\
\text { median }(\mathbf{I Q R})\end{array}$ \\
\hline Weight, $\mathrm{kg}$ & $64.2 \pm 17.7$ \\
Body surface area, $\mathrm{m}^{2}$ & $1.75 \pm 0.29$ \\
Body mass index, $\mathrm{kg} / \mathrm{m}^{2}$ & $21.4 \pm 4.0$ \\
Peak minute ventilation, $\mathrm{L} / \mathrm{min}$ & $83.4 \pm 24.1$ \\
Forced expiratory volume in first second, $\mathrm{L}$ & $3.44 \pm 0.91$ \\
Breathing reserve, $\%$ & $37(27-47)$ \\
Peak heart rate & \\
Beats/min & $184(177-192)$ \\
\% predicted & $98(94-102)$ \\
Abnormal $(\leq 85 \%), \mathrm{n}(\%)$ & $4(6)$ \\
Peak systolic blood pressure, mm Hg & $183 \pm 27$ \\
Peak workload & \\
Watt & $208 \pm 55$ \\
\% predicted & $96 \pm 19$ \\
Peak oxygen uptake & \\
mL/kg/min & \\
\% predicted & $40.1 \pm 10.8$ \\
Abnormal ( $\leq 84 \%), \mathrm{n}(\%)$ & $88 \pm 20$ \\
mL/peak heart rate & $29(42)$ \\
\% predicted & $13.6 \pm 3.9$ \\
Minute ventilation/carbon dioxide elimination & $90 \pm 18$ \\
Slope & \\
Abnormal slope $(\geq 34), \mathrm{n}(\%)$ & $23.7 \pm 3.8$ \\
Peak respiratory exchange ratio & $1.17 \pm 0.10$ \\
\hline$S D$ Standard deviation; $I O R$, interquartile range. & \\
&
\end{tabular}

was $23.7 \pm 3.8 ; 1$ patient $(1 \%)$ had an abnormal $\mathrm{VE} / \mathrm{VCO}_{2}$ slope (35).

\section{Age, Sex, and Imaging Features Related to Functional Outcome}

Univariable correlations of age, sex, and imaging features with exercise capacity and ventilatory efficiency are presented in Table 3. Age negatively correlated with $\mathrm{VO}_{2}$ peak\% $(r=-0.315, P=.008)$, and male patients generally had better exercise capacity $(r=0.318$, $P=.008)$. RV end-diastolic volume and stroke volume were both significantly associated with exercise capacity and ventilatory efficiency. MPA area significantly correlated with $\mathrm{VO}_{2}$ peak $\%(r=0.401, P=.001)$; however, the sum of the LPA and RPA area, and the smallest pulmonary branch area were not related to $\mathrm{VO}_{2}$ peak $\%$. Smallest pulmonary branch area $\left(\mathrm{mm}^{2} / \mathrm{m}^{2}\right)$ and smallest relative branch flow $(\%)$ were significantly associated with $\mathrm{VE} / \mathrm{VCO}_{2}$ slope $(r=-0.301, P=.013$ and $r=-0.307, P=.014$, respectively).

The MPA area was also significantly related to RV stroke volume $(r=0.273, P=.022)$ and $\mathrm{RV}$ systolic pressure $(r=-0.493, P=.001)$. We repeated all analyses with exclusion of patients after pulmonary artery banding, because this is usually not an accepted practice in the contemporary era. This did not yield different conclusions. 
TABLE 3. Univariable correlations of age, sex, and imaging features with exercise capacity and ventilatory efficiency in patients after arterial switch operation

\begin{tabular}{|c|c|c|}
\hline & $\mathrm{VO}_{2}$ peak $\%(\mathbf{r})$ & $\begin{array}{l}\mathrm{VE} / \mathrm{VCO}_{2} \\
\text { slope }(\mathbf{r})\end{array}$ \\
\hline Age, $\mathrm{y}_{\dagger}^{\dagger}$ & $-0.315 \dagger$ & 0.073 \\
\hline Sex, male & $0.318 \dagger$ & $-0.279 *$ \\
\hline RV systolic pressure, $\mathrm{mm} \mathrm{Hg}$ & -0.112 & -0.076 \\
\hline $\begin{array}{l}\text { Maximal velocity across pulmonary } \\
\text { valve, } \mathrm{cm} / \mathrm{s}\end{array}$ & $-0.304^{*}$ & 0.012 \\
\hline \multicolumn{3}{|l|}{ RV function } \\
\hline RV end-diastolic volume, $\mathrm{mL} / \mathrm{m}^{2} \ddagger$ & $0.305^{*}$ & $-0.306^{*}$ \\
\hline RV end-systolic volume, $\mathrm{mL} / \mathrm{m}^{2} \ddagger$ & 0.159 & -0.200 \\
\hline $\mathrm{RV}$ stroke volume, $\mathrm{mL} / \mathrm{m}^{2}$ & $0.400 \dagger$ & $-0.347 \dagger$ \\
\hline $\mathrm{RV}$ ejection fraction, $\%$ & 0.142 & 0.025 \\
\hline RV mass, $\mathrm{gr} / \mathrm{m}_{\dagger}^{2}$ & 0.208 & -0.235 \\
\hline \multicolumn{3}{|l|}{ Pulmonary artery cross-sectional areas } \\
\hline MPA area, $\mathrm{mm}^{2} / \mathrm{m}^{2}$ & $0.401 \dagger$ & -0.043 \\
\hline LPA area, $\mathrm{mm}^{2} / \mathrm{m}^{2} \ddagger$ & 0.044 & $-0.249^{*}$ \\
\hline RPA area, $\mathrm{mm}^{2} / \mathrm{m}^{2} \ddagger$ & 0.115 & -0.182 \\
\hline $\mathrm{LPA}+\mathrm{RPA}$ area, $\mathrm{mm}^{2} / \mathrm{m}^{2} \dagger$ & 0.093 & -0.233 \\
\hline $\begin{array}{l}\text { Smallest pulmonary branch area, } \\
\mathrm{mm}^{2} / \mathrm{m}^{2} \ddagger\end{array}$ & 0.109 & $-0.301 *$ \\
\hline \multicolumn{3}{|l|}{ Qflow measurements } \\
\hline LPA relative area change, $\% \ddagger$ & -0.032 & -0.178 \\
\hline RPA relative area change, $\% \ddagger$ & -0.051 & 0.152 \\
\hline LPA: RPA flow, $\%$ & $0.141:-0.141$ & $-0.329+: 0.329 \dagger$ \\
\hline $\begin{array}{l}\text { Smallest pulmonary branch } \\
\text { flow, } \% \ddagger\end{array}$ & 0.105 & $-0.307^{*}$ \\
\hline $\begin{array}{l}\text { Smallest pulmonary branch } \\
\text { flow, } \mathrm{mL} / \mathrm{m}^{2} \ddagger\end{array}$ & $0.260 *$ & $-0.264 *$ \\
\hline LPA peak flow velocity, $\mathrm{cm} / \mathrm{s}$ & -0.070 & 0.154 \\
\hline RPA peak flow velocity, $\mathrm{cm} / \mathrm{s} \ddagger$ & $-0.249^{*}$ & $0.288^{*}$ \\
\hline LPA regurgitation fraction, $\% \ddagger$ & -0.076 & -0.156 \\
\hline RPA regurgitation fraction, $\% \ddagger$ & 0.002 & 0.245 \\
\hline
\end{tabular}

$\mathrm{VO}_{2}$ peak $\%$, Exercise capacity reflected by percentage of predicted peak oxygen uptake; $\mathrm{VE} / \mathrm{VCO}_{2}$ slope, ventilatory efficiency reflected as minute ventilation relative to carbon dioxide elimination; $R V$, right ventricular; $M P A$, main pulmonary artery; $L P A$, left pulmonary artery; $R P A$, right pulmonary artery. $* P<.05 . \dagger P<.01$. $\ddagger$ †Spearman's correlation; other Pearson's correlation.

\section{Subanalysis in 3 Age Groups}

Results of the subanalysis in 3 predefined age groups $(<18,18-25$, and $>25$ years $)$ are presented in Figure 1 and Table 4. As presented in Figure 1, MPA area was not associated with exercise capacity in patients aged less than 18 years $(\beta=-0.034, P=.635)$, who had the largest MPA areas, whereas it was significantly related to exercise capacity both in patients aged 18 to 25 years and in patients aged more than 25 years $(\beta=0.134, P=.008$ and $\beta=0.219, P=.045$, respectively).

Table 4 shows the operative characteristics and imaging features per age group. Pulmonary artery banding was almost exclusively performed in the oldest age group $(P<.001)$. Patients from the earliest surgical era underwent repair at an older age $(P=.001)$, and for the reconstruction of the MPA mostly separate patches were used (vs pantaloon-type patch in younger patients) using pericardium treated with glutaraldehyde (vs untreated pericardium in younger patients). Oldest patients also had smallest MPA areas $\left(229.6 \pm 47.7 \mathrm{~mm}^{2} / \mathrm{m}^{2}, 196.3 \pm 77.7 \mathrm{~mm}^{2} / \mathrm{m}^{2}\right.$, and $\left.133.6 \pm 51.2 \mathrm{~mm}^{2} / \mathrm{m}^{2}, P<.001\right)$, highest maximal velocity across the pulmonary valve $(P=.001)$, highest $\mathrm{RV}$ mass $(P=.035)$, highest RV systolic pressures $(P<.001)$, and a trend toward a lower $\mathrm{VO}_{2}$ peak $\%(P=.051)$. Pulmonary branch areas, flow, and $\mathrm{VE} / \mathrm{VCO}_{2}$ slope were not significantly different among the 3 age groups.

\section{Lecompte Maneuver}

Pulmonary branch areas were smaller in patients who underwent the Lecompte maneuver compared with patients who did not (LPA 52 [39-75] $\mathrm{mm}^{2} / \mathrm{m}^{2}$ vs 128 [91-149] $\mathrm{mm}^{2} / \mathrm{m}^{2}, P<.001$ and RPA $89[58-139] \mathrm{mm}^{2} / \mathrm{m}^{2}$ vs 145 [109-187] $\left.\mathrm{mm}^{2} / \mathrm{m}^{2}, P=.053\right)$; however, this did not translate into an abnormal PBF distribution (mean LPA:RPA relative flow 46:54 vs $47: 53 \%, P=.181$ ). MPA area, $\mathrm{VO}_{2}$ peak $\%$, and $\mathrm{VE} / \mathrm{VCO}_{2}$ slope were comparable in both groups $(P=.971, P=.216$, and $P=.555$, respectively).

\section{Multivariable Analysis}

MPA area remained significantly associated with exercise capacity $(\beta=0.102, P=.032)$ after adjustment for age, sex, and operative characteristics (age at repair, pulmonary artery banding, patch type and treatment, RVOT reintervention). In this multivariable model, MPA area was the only significant predictor of exercise capacity.

\section{Reproducibility}

Inter- and intraobserver variability for measurement of MPA area on MRA were reflected by intraclass correlation coefficients of 0.993 and 0.997 and mean differences of $2.1 \pm 10.7 \mathrm{~mm}^{2} / \mathrm{m}^{2}(P=.564)$ and $7.3 \pm 8.0 \mathrm{~mm}^{2} / \mathrm{m}^{2}$ $(P=.019)$, respectively. Mean relative differences for inter- and intraobserver variability were $0.7 \% \pm 5.3 \%$ and $-3.7 \% \pm 3.2 \%$.

\section{DISCUSSION}

In this cross-sectional cohort study of patients long-term after arterial switch operation, both MPA and pulmonary branch areas were significantly decreased compared with healthy controls, and abnormal exercise capacity was common $(42 \%)$. Cross-sectional MPA area was independently associated with exercise capacity in patients aged more than 18 years and correlated with stroke volume at rest and RV systolic pressure.

Significant unilateral pulmonary branch stenosis was present in $37 \%$ of patients; however, this resulted in abnormal blood flow distribution in only $7 \%$ of patients. Other factors that could inhibit exercise capacity in this group of patients, such as RV dysfunction or chronotropic 

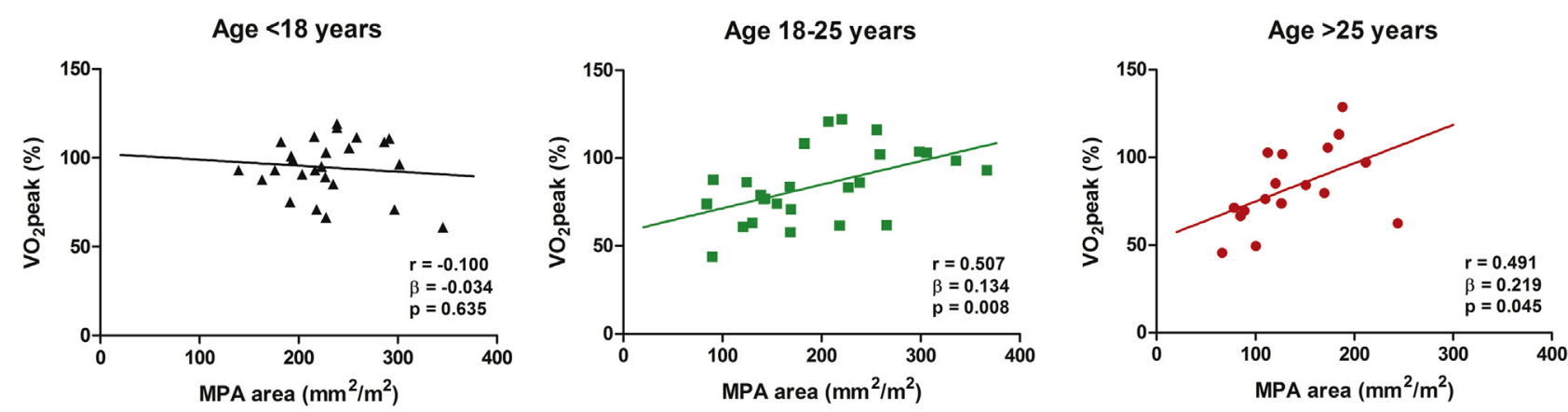

FIGURE 1. MPA area is not associated with exercise capacity in patients aged less than 18 years, who have the largest MPA areas $(\beta=-0.034, P=.635)$, whereas it is significantly related to exercise capacity in patients aged 18 to 25 years and more than 25 years $(\beta=0.134, P=.008$ and $\beta=0.219, P=.045$, respectively). $\mathrm{VO}_{2}$ peak $\%$, Exercise capacity reflected by percentage of predicted peak oxygen uptake; $M P A$, main pulmonary artery.

incompetence, were uncommon in our cohort. These results indicate that narrowing of the MPA is a common finding in adult patients after the arterial switch operation and that it is the main right-sided lesion limiting functional capacity, rather than RV dysfunction or unilateral pulmonary branch stenosis.

Although abnormal PBF distribution was uncommon in our cohort, smallest relative pulmonary branch flow was associated with ventilatory efficiency. This was also reported by Giardini and colleagues, ${ }^{7}$ who identified abnormal PBF distribution as an independent predictor of both decreased exercise capacity and ventilatory efficiency. In contrast to our results, abnormal PBF distribution was more common in their study (18\%) and MPA area was not an independent predictor of exercise capacity. This is probably explained by the younger age of the patient group

TABLE 4. Subanalysis for operative characteristics, imaging features, and functional outcome among 3 age groups

\begin{tabular}{|c|c|c|c|c|}
\hline Variable & $<18$ y $(n=25)$ & $18-25$ y $(n=26)$ & $>25$ y $(n=20)$ & $P$ value \\
\hline Sex, male $\mathrm{n}(\%) \ddagger$ & $18(72)$ & $20(77)$ & $14(70)$ & .910 \\
\hline \multicolumn{5}{|l|}{ Operative characteristics } \\
\hline Rashkind procedure, $\mathrm{n}(\%) \ddagger$ & $22(88)$ & $14(54)$ & $12(60)$ & .089 \\
\hline Pulmonary artery banding, $\mathrm{n}(\%) \ddagger$ & $0(0)$ & $1(4)$ & $7(35)$ & $<.001 \dagger$ \\
\hline Age at surgical repair, d & $9.2 \pm 8.8$ & $15.5 \pm 26.9$ & $125.8 \pm 201.2$ & $.001 \dagger$ \\
\hline Pantaloon-type patch, n (\%) & $23(92)$ & $10(40 \S)$ & $1(8 \|)$ & $<.001 \dagger$ \\
\hline Untreated pericardium, $\mathrm{n}(\%)$ & $25(100)$ & $14(56 \S)$ & $0(0 \|)$ & $<.001 \dagger$ \\
\hline Lecompte maneuver, $\mathrm{n}(\%) \ddagger$ & $23(92)$ & $23(89)$ & $17(85)$ & .463 \\
\hline Ventricular septal defect repair, n $(\%) \ddagger$ & $5(20)$ & $8(31)$ & $7(35)$ & .260 \\
\hline Cardiopulmonary bypass time, min & $153 \pm 59$ & $207 \pm 53$ & $187 \pm 45$ & .104 \\
\hline Crossclamp time, $\min$ & $92 \pm 18$ & $125 \pm 34$ & $106 \pm 27$ & .171 \\
\hline RVOT reintervention, $\mathrm{n}(\%) \ddagger$ & $0(0)$ & $2(8)$ & $9(45)$ & $<.001 \dagger$ \\
\hline \multicolumn{5}{|l|}{ Imaging features } \\
\hline $\mathrm{RV}$ systolic pressure, $\mathrm{mm} \mathrm{Hg}$ & $22.7 \pm 9.6$ & $29.2 \pm 6.8$ & $36.5 \pm 9.9$ & $<.001 \dagger$ \\
\hline Maximal velocity across pulmonary valve, $\mathrm{cm} / \mathrm{s}$ & $183.7 \pm 58.0$ & $194.5 \pm 72.4$ & $254.6 \pm 52.7$ & $.001 \dagger$ \\
\hline $\mathrm{RV}$ end-diastolic volume, $\mathrm{mL} / \mathrm{m}^{2}$ & $94.1 \pm 12.0$ & $96.9 \pm 12.4$ & $94.5 \pm 23.7$ & .909 \\
\hline $\mathrm{RV}$ stroke volume, $\mathrm{mL} / \mathrm{m}^{2}$ & $51.7 \pm 8.3$ & $53.3 \pm 7.0$ & $52.6 \pm 10.5$ & .690 \\
\hline $\mathrm{RV}$ mass, $\mathrm{g} / \mathrm{m}^{2}$ & $29.6 \pm 4.7$ & $30.6 \pm 6.0$ & $34.1 \pm 9.5$ & $.035 *$ \\
\hline MPA area, $\mathrm{mm}^{2} / \mathrm{m}^{2}$ & $229.6 \pm 47.7$ & $196.3 \pm 77.7$ & $133.6 \pm 51.2$ & $<.001 \dagger$ \\
\hline $\mathrm{LPA}+\mathrm{RPA}$ area, $\mathrm{mm}^{2} / \mathrm{m}^{2}$ & $171.0 \pm 55.5$ & $176.2 \pm 81.4$ & $184.5 \pm 102.1$ & .581 \\
\hline Smallest pulmonary branch area, $\mathrm{mm}^{2} / \mathrm{m}^{2}$ & $57.7 \pm 28.9$ & $58.3 \pm 35.0$ & $67.6 \pm 44.7$ & .388 \\
\hline Smallest pulmonary branch flow, $\mathrm{mL} / \mathrm{m}^{2}$ & $22.0 \pm 4.5$ & $21.0 \pm 4.0$ & $21.2 \pm 4.3$ & .494 \\
\hline Smallest pulmonary branch flow, \% & $43.7 \pm 6.0$ & $41.7 \pm 5.9$ & $42.5 \pm 5.0$ & .435 \\
\hline \multicolumn{5}{|l|}{ Functional outcome } \\
\hline $\mathrm{VO}_{2}$ peak, $\%$ & $94.7 \pm 16.1$ & $84.4 \pm 20.6$ & $83.4 \pm 21.9$ & .051 \\
\hline $\mathrm{VE} / \mathrm{VCO}_{2}$ slope & $23.0 \pm 2.9$ & $24.3 \pm 3.6$ & $24.0 \pm 5.1$ & .379 \\
\hline
\end{tabular}

$R V O T$, Right ventricular outflow tract; $R V$, right ventricular; $M P A$, main pulmonary artery; $L P A$, left pulmonary artery; $R P A$, right pulmonary artery; $V O_{2} p e a k \%$, exercise capacity reflected by percentage of predicted peak oxygen uptake; $V E / V C \mathrm{O}_{2}$ slope, ventilatory efficiency reflected as minute ventilation relative to carbon dioxide elimination. $* P<.05$. $\dagger P<.01$. $\ddagger$ n $(\%)$ and chi-square Mantel-Haenszel test, other mean \pm standard deviation and univariable linear regression. $\S$ Data available in 25 of 26 patients. $\|$ Data available in 12 of 20 patients. 
in their study (13.3 \pm 3.4 years), because MPA area was not related to exercise capacity in the youngest patients of our cohort.

\section{Limitation of Stroke Volume Increase During Exercise}

In normal subjects, the RV stroke volume increases during exercise with only mild elevation of RV pressures. This is a consequence of low pulmonary vascular resistance, mediated by recruitment of closed vessels and distension of already opened vessels. ${ }^{18,19}$ We found that MPA area significantly correlated with RV stroke volume at rest and RV systolic pressure. This may imply that in patients with pulmonary artery narrowing, RV systolic pressure increases disproportionately during exercise, limiting the increase in RV stroke volume during exercise, subsequently limiting exercise capacity.

\section{Relative Area Change}

Decreased distensibility of the MPA or branches has been proposed as a possible mechanism for reduced exercise capacity in patients after the arterial switch operation. Grotenhuis and colleagues ${ }^{20}$ and Voges and colleagues ${ }^{21}$ demonstrated a reduced distensibility of the proximal aorta in these patients. This was potentially mediated by increased aortic wall stress of the dilated root, fibrous tissue, and intrinsic wall abnormalities near the suture line and pulmonary artery branches embracing the aorta after the Lecompte maneuver. ${ }^{17}$ Most of these features could theoretically also affect distensibility of the MPA and branches, contributing to a functional stenosis on exercise. ${ }^{7,22}$ This study approximated distensibility of the pulmonary branches by relative area change, which is uncorrected for pulse pressure, because our study protocol did not include invasive measurements. To our knowledge, these measurements have not been performed in patients after the arterial switch operation. Our data do not provide evidence for a decreased relative area change of the LPA and RPA. However, relative area change of the MPA was not assessed in this study and could be altered because of different elastic properties of the pericardial patch.

\section{Smaller Main Pulmonary Artery Areas in Older Age Groups}

Patients aged more than 18 years had smaller MPA areas compared with younger patients. Because this is a crosssectional study, it is uncertain whether this diminution of size is a reflection of learning curve and changing surgical techniques over time or the result of rapid somatic growth with inadequate growth at the anastomotic site, as suggested by Prifti and colleagues. ${ }^{23}$ The operative characteristics of patients aged more than 25 years showed clear differences with both other age categories. Pulmonary artery banding was almost exclusively performed in this age group; patients were markedly older at time of surgical repair, and for the reconstruction of the MPA, mostly separate patches of pericardium treated with glutaraldehyde were used (vs untreated pantaloon type patch). Single pantaloon-type patch has been reported to result in less residual RVOT obstruction, and, in contrast to treated pericardium, untreated pericardial tissue potentially retains the ability to grow and adapt. ${ }^{23}$ Therefore, pulmonary artery banding and type of MPA reconstruction (separate patches, treated pericardium with inadequate growth) are probably important causes of MPA narrowing.

Prolonged cyanosis and low PBF in patients who underwent the arterial switch operation at an older age also may adversely affect the microscopic pulmonary vasculature, myocardium, and systemic circulation, leading to a reduced $\mathrm{VO}_{2}$ peak $\%$.

By using multivariable linear regression, we adjusted for the effects of age, sex, and operative characteristics (age at repair, pulmonary artery banding, patch type and treatment, RVOT reintervention). In multivariable analysis, MPA area was the only significant predictor of exercise capacity.

\section{Clinical Implications}

In this study, the importance of MPA narrowing as a determinant for exercise capacity is highlighted. This could imply that during the arterial switch operation, optimization of MPA size is important for functional outcomes. Moreover, this suggests that the long-term follow-up of patients after the arterial switch operation should focus on the presence or progression of MPA stenosis. Pulmonary branch reinterventions in patients with unilateral pulmonary branch stenosis might only improve functional capacity in the small subgroup of patients with an abnormal PBF distribution. This hypothesis requires further investigation in longitudinal studies.

\section{Study Limitations}

Limitations of this study are inherent to its design, because this is a single-center, cross-sectional study. Therefore, variance in operation procedures and surgical experience among surgical centers could result in different outcomes in other patient cohorts. Second, we need longitudinal follow-up studies to assess the actual impact of RVOT reinterventions on functional outcome in adult patients after the arterial switch operation. Individual follow-up data should elucidate whether the observation of decreased MPA areas in older age groups can be partially explained by rapid somatic growth. Finally, patients and controls were not perfectly matched, because patients were younger than healthy controls and included more male patients. To account for this, all measurements were indexed for body surface area. Moreover, because patients aged less than 18 years exhibited the smallest differences 
compared with healthy controls and male patients generally had better exercise capacity, age and sex matching would have only strengthened the differences found in this study.

\section{CONCLUSIONS}

In this cross-sectional study of patients long-term after the arterial switch operation, the MPA area was increasingly smaller in older age groups. The MPA area was significantly related to exercise capacity, independently of age, sex, and operative characteristics. Although branch pulmonary artery areas were smaller than in healthy controls, PBF at rest and ventilatory efficiency were generally not compromised. Therefore, our data suggest that MPA narrowing is an important and thus far underappreciated determinant in the limitation of functional capacity in adults after the arterial switch operation.

\section{Conflict of Interest Statement}

Authors have nothing to disclose with regard to commercial support.

\section{References}

1. Centers for Disease Control and Prevention. Improved national prevalence estimates for 18 selected major birth defects - United States, 1999-2001. MMWR Morb Mortal Wkly Rep. 2006;54:1301-5.

2. Jatene AD, Fontes VF, Paulista PP, Souza LC, Neger F, Galantier M, et al. Anatomic correction of transposition of the great vessels. J Thorac Cardiovasc Surg. 1976;72:364-70.

3. Angeli E, Raisky O, Bonnet D, Sidi D, Vouhe PR. Late reoperations after neonatal arterial switch operation for transposition of the great arteries. Eur J Cardiothorac Surg. 2008;34:32-6.

4. Khairy P, Clair M, Fernandes SM, Blume ED, Powell AJ, Newburger JW, et al. Cardiovascular outcomes after the arterial switch operation for D-transposition of the great arteries. Circulation. 2013;127:331-9.

5. Fredriksen PM, Pettersen E, Thaulow E. Declining aerobic capacity of patients with arterial and atrial switch procedures. Pediatr Cardiol. 2009;30:166-71.

6. Giardini A, Khambadkone S, Rizzo N, Riley G, Napoleone CP, Muthialu N, et al. Determinants of exercise capacity after arterial switch operation for transposition of the great arteries. Am J Cardiol. 2009;104:1007-12.

7. Giardini A, Khambadkone S, Taylor A, Derrick G. Effect of abnormal pulmonary flow distribution on ventilatory efficiency and exercise capacity after arterial switch operation for transposition of great arteries. Am J Cardiol. 2010;106: 1023-8.

8. Villafañe J, Lantin-Hermoso MR, Bhatt AB, Tweddell JS, Geva T, Nathan M, et al. D-transposition of the great arteries: the current era of the arterial switch operation. J Am Coll Cardiol. 2014;64:498-511.
9. American Thoracic Society/American College of Chest Physicians. ATS/ACCP Statement on cardiopulmonary exercise testing. Am J Respir Crit Care Med. 2003;167:211-77.

10. Lotz J, Meier C, Leppert A, Galanski M. Cardiovascular flow measurement with phase-contrast MR Imaging: basic facts and implementation. Radiographics. 2002;22:651-71.

11. Driessen MM, Baggen VJ, Freling HG, Pieper PG, van Dijk AP, Doevendans PA, et al. Pressure overloaded right ventricles: a multicenter study on the importance of trabeculae in RV function measured by CMR. Int J Cardiovasc Imaging. 2014; 30:599-608.

12. Freling HG, van Wijk K, Jaspers K, Pieper PG, Vermeulen KM, van Swieten JM et al. Impact of right ventricular endocardial trabeculae on volumes and function assessed by CMR in patients with tetralogy of Fallot. Int J Cardiovasc Imaging. 2012;29:625-31

13. Merkx MA, Bescós JO, Geerts L, Bosboom EM, van de Vosse FN, Breeuwer M Accuracy and precision of vessel area assessment: manual versus automatic lumen delineation based on full-width at half-maximum. J Magn Reson Imaging. 2012;36:1186-93.

14. Godfrey S. Exercise Testing in Children. London: W.B. Saunders Company 1974.

15. Wasserman K, Sue D. Principles of Exercise Testing and Interpretation. 2nd ed. Philadelphia: Lea \& Febiger; 1994.

16. Ten Harkel AD, Takken T, Van Osch-Gevers M, Helbing WA. Normal values for cardiopulmonary exercise testing in children. Eur J Cardiovasc Prev Rehabil. 2011;18:48-54.

17. Lecompte Y, Zannini L, Hazan E, Jarreau MM, Bex JP, Tu TV, et al. Anatomic correction of transposition of the great arteries. J Thorac Cardiovasc Surg. 1981;82:629-31.

18. Kovacs G, Berghold A, Scheidl S, Olschewski H. Pulmonary arterial pressure during rest and exercise in healthy subjects: a systematic review. Eur Respir J. 2009;34:888-94.

19. Naeije R, Chesler N. Pulmonary circulation at exercise. Compr Physiol. 2012;2: 711-41.

20. Grotenhuis HB, Ottenkamp J, Fontein D, Vliegen HW, Westenberg JJ, Kroft LJ, et al. Aortic elasticity and left ventricular function after arterial switch operation: MR imaging-initial experience. Radiology. 2008;249:801-9.

21. Voges I, Jerosch-Herold M, Hedderich J, Hart C, Petko C, Scheewe J, et al. Implications of early aortic stiffening in patients with transposition of the great arteries after arterial switch operation. Circ Cardiovasc Imaging. 2013;6: 245-53.

22. Gutberlet M, Boeckel T, Hosten N, Vogel M, Kühne T, Oellinger H, et al. Arterial switch procedure for D-transposition of the great arteries: quantitative midterm evaluation of hemodynamic changes with cine MR imaging and phase-shift velocity mapping-initial experience. Radiology. 2000;214: 467-75.

23. Prifti E, Crucean A, Bonacchi M, Bernabei M, Murzi B, Luisi SV, et al. Early and long term outcome of the arterial switch operation for transposition of the great arteries: predictors and functional evaluation. Eur J Cardiothorac Surg. 2002;22: 864-73.

Key Words: transposition of great vessels, arterial switch operation, magnetic resonance imaging, exercise capacity, pulmonary artery stenosis 